Functiones et Approximatio

54.1 (2016), 57-63

doi: $10.7169 / \mathrm{facm} / 2016.54 .1 .5$

\title{
MULTIPLES OF SQUARES IN SHORT INTERVALS
}

\author{
JoËL Rivat, IgOR E. SHPARLINSKI
}

\begin{abstract}
We use the theory of exponent pairs and Vaaler polynomials to show that any interval of the form $\left[x, x+x^{1 / 2}\right]$ contains an integral multiple $m^{2} r \in\left[x, x+x^{1 / 2}\right]$ of a perfect square $m^{2}$ with an integer $m>x^{0.281286}$.
\end{abstract}

Keywords: multiples of squares, short intervals, exponential sums.

\section{Introduction}

Clearly any interval of the form $\left[x, x+2 x^{1 / 2}+1\right]$ with $x \geqslant 1$ contains a perfect integer square, while some shorter intervals do not. Furthermore, any interval of the form $[x, x+y]$ with real positive $x$ and $y$ contains an integral multiple $m^{2} r$ of a perfect square $m^{2}$ with a positive integer $m \leqslant\left\lfloor y^{1 / 2}\right\rfloor$.

Here, in a wide range of relative sizes of sufficiently large positive $x$ and $y$ we show that any interval of the form $[x, x+y]$ contains an integral multiple $m^{2} r$ of a perfect square $m^{2}$ with an integer $m=y^{\gamma+o(1)}$ with some fixed $\gamma>1 / 2$. In particular, in the most interesting "borderline" case when $y=x^{1 / 2+o(1)}$ one can take $\gamma=346 / 615=0.5626 \ldots$

Our main tool is the theory of exponent pairs, we refer to [1, Chapter 3], [3, Sections 7.3 and 17.4], [5, Chapter 8] and [6, Chapter 3] for an exact definition, properties and examples of exponent pairs. Note that in the terminology of [5], which we accept here, an exponent pair $(\kappa, \lambda)$ corresponds to the exponent pair $(\kappa, \lambda+1 / 2)$ in the terminology of $[1,3,6]$.

Throughout the paper, any implied constants in symbols $O$ and $\ll$ may depend on the real positive parameter $\varepsilon$ and are absolute otherwise. We recall that the notations $U=O(V)$ and $U \ll V$ are all equivalent to the statement that $|U| \leqslant c V$ holds with some constant $c>0$. We also use $U \asymp V$ to denote that $U \ll V \ll U$. For a real $A \geqslant 1$ and an integer $a$ we write $a \sim A$ to express that $A \leqslant a \leqslant 2 A$.

2010 Mathematics Subject Classification: primary: 11N25 
Theorem 1. For real numbers $x$ and $y$ with $x^{1 / 2}>y>1$ the interval $[x, x+y]$ contains an integral multiple $m^{2} r \in[x, x+y]$ of a perfect square $m^{2}$ with an integer

$$
m=x^{-\alpha+o(1)} y^{\beta},
$$

as $x \rightarrow \infty$, provided that $x^{\alpha} \leqslant y^{\beta}$, where

$$
\alpha=\frac{2 \kappa}{3+2 \lambda-2 \kappa} \quad \text { and } \quad \beta=\frac{2+2 \kappa}{3+2 \lambda-2 \kappa},
$$

for an arbitrary exponent pair $(\kappa, \lambda)$.

We now immediately derive:

Corollary 2. For an integer $N \geqslant 1$, for any fixed $\vartheta$ with $\alpha / \beta \leqslant \vartheta \leqslant 1$ there are integers

$$
1 \leqslant r \leqslant N^{1-2 \beta \vartheta+2 \alpha+o(1)} \quad \text { and } \quad 0 \leqslant s \leqslant N^{\vartheta}
$$

as $N \rightarrow \infty$, such that $r(N+s)$ is a perfect square, where $\alpha$ and $\beta$ are as in Theorem 1.

Indeed, we apply Theorem 1 with $x=N$ and $y=N^{\vartheta}$, thus the condition $x^{\alpha} \leqslant y^{\beta}$ is satisfied. Then, for $m$ and $r$ as in Theorem 1 , we define $s$ by the condition $s=m^{2} r-N$, thus $r(N+s)=(m r)^{2}$. Furthermore, we have

$$
x-N=0 \leqslant s \leqslant x+y-N=N^{\vartheta}
$$

and

$$
1 \leqslant r \leqslant\left(x+x^{\vartheta}\right) / m^{2} \ll x / m^{2}=x\left(x^{-\alpha+o(1)} y^{\beta}\right)^{-2}=N^{1-2 \beta \vartheta+2 \alpha+o(1)} .
$$

Clearly, the result of Corollary 2 is nontrivial only for $\vartheta \leqslant 1 / 2$.

We remark that a somewhat related question about multiples of squares in short intervals, motivated by application to computer arithmetic, has been studied by Hanrot, Rivat, Tenenbaum and Zimmermann [2] via similar techniques.

\section{Fractional parts and exponential sums}

As usual, we define the function $\psi(u)=u-\lfloor u\rfloor-1 / 2$, where $\lfloor u\rfloor$ is the integer part of a real $u$. By a result of Vaaler [7], see also [1, Theorem A.6].

Lemma 3. For any integer $H \geqslant 1$ there is a trigonometric polynomial

$$
\psi_{H}(u)=\sum_{1 \leqslant|h| \leqslant H} \frac{a_{h}}{-2 i \pi h} \mathbf{e}(h u)
$$

for coefficients $a_{h} \in[0,1]$ and such that

$$
\left|\psi(u)-\psi_{H}(u)\right| \leqslant \frac{1}{2 H+2} \sum_{|h| \leqslant H}\left(1-\frac{|h|}{H+1}\right) \mathbf{e}(h u) .
$$


As we have mentioned we combine Lemma 3 with the bounds of exponential sums based on the theory of exponent pairs, see [1, Chapter 3] or [5, Chapter 8]. In our case we apply it to the monomial functions $f(z)=A / z^{2}$ with $A \geqslant 1$ so that the condition $\left[5\right.$, Equation (8.55)] is satisfied (with $F=A / N^{2}$ ). More precisely, we have:

Lemma 4. Let $(\kappa, \lambda)$ be an exponent pair. For any $A \geqslant M \geqslant N \geqslant 1$ with $N \sim M$ and such that $A \geqslant M^{3}$, we have

$$
\sum_{M \leqslant m<N} \mathbf{e}\left(\frac{A}{m^{2}}\right) \ll A^{\kappa+o(1)} M^{1 / 2+\lambda-3 \kappa}
$$

where the implied constant depends only on $\kappa$ and $\lambda$.

Proof. First we note that for $f(z)=A / z^{2}$ on the interval $z \in[M, 2 M]$ we have

$$
F / M^{-j} \ll\left|f^{(j)}(z)\right| \ll F / M^{-j}
$$

where $F=A / M^{2}$. Furthermore, for $\Lambda=F M^{-1}=A / M^{-3}$ we have $\Lambda \geqslant 1$, so the condition [5, Equation (8.56)] holds and thus by [5, Equation (8.55)] we have

$$
\sum_{M \leqslant m<N} \mathbf{e}\left(\frac{A}{m^{2}}\right) \ll \Lambda^{\kappa} M^{1 / 2+\lambda} A^{o(1)} .
$$

After simple calculations we obtain the result.

We also recall that given an exponent pair $(\kappa, \lambda)$ one can produce a series of other pairs by applying, the so-called $\mathcal{A}$ - and $\mathcal{B}$-processes, which in the terminology of $[5$, Section 8.4$]$ can be written as

$$
\mathcal{A}(\kappa, \lambda)=\left(\frac{\kappa}{2 \kappa+2}, \frac{\lambda+1 / 2}{2 \kappa+2}\right) \quad \text { and } \quad \mathcal{B}(\kappa, \lambda)=(\lambda, \kappa)
$$

\section{Proof of Theorem 1}

For $1 \leqslant y \leqslant x$ and $M \in \mathbb{N}$ we consider the sum

$$
S(x, y, M)=\sum_{m \sim M}\left(\left\lfloor\frac{x+y}{m^{2}}\right\rfloor-\left\lfloor\frac{x}{m^{2}}\right\rfloor\right) .
$$

Clearly, the positivity $S(x, y, M)>0$ implies that there is $m \sim M$ with

$$
m^{2}\left(\left\lfloor\frac{x}{m^{2}}\right\rfloor+1\right) \in[x, x+y] .
$$

Using the function $\psi(u)$ we write

$$
S(x, y, M)=\sum_{m \sim M} \frac{y}{m^{2}}-\sum_{m \sim M}\left(\psi\left(\frac{x+y}{m^{2}}\right)-\psi\left(\frac{x}{m^{2}}\right)\right) .
$$


Using the Vaaler polynomials given in Lemma 3, for any integer $H \geqslant 1$ we can write

$$
\left|S(x, y, M)-y \sum_{m \sim M} \frac{1}{m^{2}}\right| \leqslant E(x+y, M, H)+E(x, M, H)+|F(x, y, M, H)|,
$$

where

$$
E(z, M, H)=\sum_{m \sim M}\left|\psi\left(\frac{z}{m^{2}}\right)-\psi_{H}\left(\frac{z}{m^{2}}\right)\right|
$$

and

$$
F(x, y, M, H)=\sum_{m \sim M}\left(\psi_{H}\left(\frac{x+y}{m^{2}}\right)-\psi_{H}\left(\frac{x}{m^{2}}\right)\right) .
$$

We see from Lemma 3 that

$$
E(z, M, H) \leqslant \frac{M}{2 H+2}+\frac{1}{2 H+2} \sum_{1 \leqslant|h| \leqslant H}\left(1-\frac{|h|}{H+1}\right) \sum_{m \sim M} \mathbf{e}\left(\frac{h z}{m^{2}}\right)
$$

and also

$$
F(x, y, M, H)=\sum_{1 \leqslant|h| \leqslant H} \frac{a_{h}}{-2 i \pi h} \sum_{m \sim M}\left(\mathbf{e}\left(\frac{h y}{m^{2}}\right)-1\right) \mathbf{e}\left(\frac{h x}{m^{2}}\right) .
$$

We have

$$
\begin{aligned}
\left(\mathbf{e}\left(\frac{h y}{(m+1)^{2}}\right)-1\right)-\left(\mathbf{e}\left(\frac{h y}{m^{2}}\right)-1\right) & =\mathbf{e}\left(\frac{h y}{(m+1)^{2}}\right)-\mathbf{e}\left(\frac{h y}{m^{2}}\right) \\
& =\mathbf{e}\left(\frac{h y}{m^{2}}\right)\left(\mathbf{e}\left(\frac{h y}{(m+1)^{2}}-\frac{h y}{m^{2}}\right)-1\right) \\
& =\mathbf{e}\left(\frac{h y}{m^{2}}\right)\left(\mathbf{e}\left(\frac{-h y(2 m+1)}{m^{2}(m+1)^{2}}\right)-1\right) \\
& =O\left(\frac{|h| y}{M^{3}}\right) .
\end{aligned}
$$

Hence, by partial summation, we derive from (3) that

$$
F(x, y, M, H) \ll \frac{y}{M^{2}} \sum_{1 \leqslant|h| \leqslant H} \max _{N \sim M}\left|\sum_{M \leqslant m<N} \mathbf{e}\left(\frac{h x}{m^{2}}\right)\right| .
$$

So, assuming that

$$
M \leqslant x^{1 / 3},
$$

we see from $(2),(4)$ and Lemma 4 that for any exponent pair $(\kappa, \lambda)$ we have

$$
S(x, y, M)-\frac{y}{2 M} \ll \frac{M}{H}+\left(\frac{1}{H}+\frac{y}{M^{2}}\right) \sum_{1 \leqslant|h| \leqslant H}(|h| x)^{\kappa} M^{1 / 2+\lambda-3 \kappa} .
$$


Let us assume that we are in the interesting case $M \geqslant y^{1 / 2}$. We then fix some sufficiently small $\varepsilon>0$ and set

$$
H=\left\lceil\frac{M^{2}}{y} x^{\varepsilon}\right\rceil .
$$

We then obtain

$$
\begin{aligned}
S(x, y, M)-\frac{y}{2 M} & \ll \frac{y}{M} x^{-\varepsilon}+x^{(1+\kappa) \varepsilon}\left(\frac{x}{y}\right)^{\kappa} M^{1 / 2+\lambda-\kappa} \\
& \ll \frac{y}{M} x^{-\varepsilon}+x^{2 \varepsilon}\left(\frac{x}{y}\right)^{\kappa} M^{1 / 2+\lambda-\kappa} .
\end{aligned}
$$

So we see that

$$
S(x, y, M)-\frac{y}{2 M} \ll \frac{y}{M} x^{-\varepsilon}
$$

under the following sufficient condition:

$$
\left(x y^{-1}\right)^{\kappa} M^{1 / 2+\lambda-\kappa} \ll \frac{y}{M} x^{-3 \varepsilon}
$$

that is,

$$
M^{3 / 2+\lambda-\kappa} \ll x^{-\kappa} y^{1+\kappa} x^{-2 \varepsilon} .
$$

Since $\varepsilon>0$ is arbitrary, we can take

$$
M=x^{-\alpha+o(1)} y^{\beta}
$$

to guarantee that $S(x, y, M)>0$.

It remains to note that for the above choice of $M$, for $y \leqslant x^{1 / 2}$ we have

$$
M \leqslant x^{-\alpha+\beta / 2+o(1)}=x^{(1-\kappa) /(3+2 \lambda-2 \kappa)+o(1)} .
$$

As we always have $\lambda, \kappa>0$, the condition (5) holds and the result now follows.

\section{Comments}

Clearly, Theorem 1 improves the trivial result with $m \asymp y^{1 / 2}$ provided that

$$
y>x^{4 \kappa /(1+6 \kappa-2 \lambda)+\varepsilon}
$$

for some fixed $\varepsilon$.

For $y=x^{1 / 2+o(1)}$ the result of Theorem 1 becomes

$$
m=x^{(1-\kappa) /(3+2 \lambda-2 \kappa)+o(1)} .
$$

Thus, for the classical exponent pair $(\kappa, \lambda)=(1 / 6,1 / 6)$, which can be obtained from the pair $(1 / 2,0)$ given by [5, Corollay 8.13$]$ with the help of the transformations (1) as $\mathcal{A B}(0,1 / 2)=(1 / 6,1 / 6)$ we obtain $m=x^{5 / 18+o(1)}$. Alternatively, the 
same value of $m$ can also be obtained for $(\kappa, \lambda)=(2 / 7,1 / 14)=\mathcal{B A}^{2} \mathcal{B}(0,1 / 2)$. Notice that for using $(\kappa, \lambda)=(1 / 6,1 / 6)$ it is sufficient to apply [1, Theorem 2.9], thus avoiding the use of the theory of exponent pairs. However using more complicated exponent pairs one can get slightly better results. For example, the pair $(9 / 56,9 / 56)$, see [5, Equation (8.68)] (which cannot be obtained from $(0,1 / 2)$ via the $\mathcal{A}$ - and $\mathcal{B}$-processes $)$ gives $m=x^{47 / 168+o(1)}$, while $(2 / 9,1 / 9)=$ $\mathcal{B} \mathcal{A B} \mathcal{B} \mathcal{B} \mathcal{A}^{2} \mathcal{B}(0,1 / 2)$ gives $m=x^{7 / 25+o(1)}$. Furthermore, with a more complicated the choice

$$
(\kappa, \lambda)=\left(\frac{32}{205}, \frac{32}{205}\right)
$$

given by Huxley [4] for $y=x^{1 / 2+o(1)}$ we obtain

$$
m=x^{173 / 615+o(1)} .
$$

Note that the numerical differences between these estimates are really minor

$$
\begin{aligned}
\frac{5}{18} & =0.2777 \ldots, & \frac{47}{168} & =0.2797 \ldots, \\
\frac{7}{25} & =0.2800 \ldots, & \frac{173}{615} & =0.2813 \ldots .
\end{aligned}
$$

It is quite possible that some other choices of $(\kappa, \lambda)$ lead to a stronger bound. In fact, the algorithm given in [1, Chapter 5] may lead to an optimal choice within the pairs obtained via the $\mathcal{A}$ - and $\mathcal{B}$-processes; we however do not expect any substantial numerical improvements. Indeed, by [6, Conjecture 2, Chapter 3], $(\varepsilon, \varepsilon)$ is an exponent pair for any $\varepsilon>0$. This leads to $m=x^{1 / 3+o(1)}$ which is the limit of our approach.

Clearly, for any sufficiently large $z$ there are at most

$$
\sum_{m \geqslant M} \frac{z}{m^{2}} \ll z / M
$$

multiples of squares $m^{2} r \leqslant z$. Thus for any $y<z^{1 / 2}$ there is $x \in[z / 2, z]$ such that the interval $[x, x+y]$ does not contain a multiple of $m^{2}$ for any $m \geqslant c y$, where $c>0$ is an absolute constant.

We also remark, that taking

$$
\vartheta=\frac{1+2 \alpha}{1+2 \beta}
$$

in Corollary 2 balances the bounds on $r$ and $s$ and with the choice (6) leads to the bound $r, s \leqslant N^{743 / 1563+o(1)}$. This also suggests the following:

Question 5. Given an integer N, obtain an upper bound on smallest values of positive integers $u$ and $v$ such that the linear transformation $u N+v$ is a perfect square. 
Note that Question 5 can be reformulated as a question about finding a bound $U$ as a function of $N$ so the congruence

$$
m^{2} \equiv v \quad(\bmod N)
$$

has a solution in positive integers $m \leqslant \sqrt{N U}$ and $v \leqslant U$. Using bounds of incomplete Gaussian sums, it is easy to show that one can take $U=N^{2 / 3+o(1)}$. It is certainly interesting to improve this bound.

We note that the same method works for multiples of higher powers. It can also be extended to multiples of many other functions such as, for example, $m(m+1)$. This flexibility of our approach may also be its main weakness as it does not use the arithmetic structure of squares.

Furthermore, it is not clear how to obtain a nontrivial result for the polynomial analogue of our question. Namely, given a monic polynomial $f \in \mathbb{F}_{q}[X]$ of degree $d$ over a finite field $\mathbb{F}_{q}$ of $q$ elements and a positive integer $e$, we ask what is the largest degree $m$ of a polynomial $g$ such that $\operatorname{deg}\left(f-g^{2} h\right) \leqslant e$ for some $h \in \mathbb{F}_{q}[X]$.

\section{References}

[1] S.W. Graham and G. Kolesnik, Van der Corput's method of exponential sums, Cambridge Univ. Press, 1991.

[2] G. Hanrot, J. Rivat, G. Tenenbaum and P. Zimmermann, Density results on floating-point invertible numbers, Theoret. Comput. Sci. 291 (2003), 135-141.

[3] M.N. Huxley, Area, lattice points and exponential sums, Oxford Univ. Press, 1996.

[4] M.N. Huxley, Exponential sums and the Riemann zeta function, V, Proc. London Math. Soc. 90 (2005), no. 1, 1-41.

[5] H. Iwaniec and E. Kowalski, Analytic number theory, Amer. Math. Soc., Providence, RI, 2004.

[6] H.L. Montgomery, Ten lectures on the interface between analytic number theory and harmonic analysis, Amer. Math. Soc., Providence, RI, 1994.

[7] J.D. Vaaler, Some extremal functions in Fourier analysis, Bull. Amer. Math. Soc. 12 (1985) 183-215.

Addresses: Joël Rivat: Institut de Mathématiques de Marseille, Université d'Aix-Marseille, 13288 Marseille, France;

Igor E. Shparlinski: Department of Pure Mathematics, University of New South Wales, Sydney, NSW 2052, Australia.

E-mail: joel.rivat@univ-amu.fr, igor.shparlinski@unsw.edu.au

Received: 19 April 2015; revised: 14 May 2015 